Ann. Parasitol. Hum. Comp., 1990, 65: $\mathrm{n}^{\circ} 3,111-125$.

Mémoire.
Key-words: Leishmania. Identification. Taxonomy. Intrinsic and extrinsic characters. Isoenzymes. Phenogram. Dendrogram.

Mots-clés : Leishmania. Identification. Caractères intrinsèques et extrinsèques. Isoenzymes. Phénogramme. Cladogramme. Révision taxonomique.

\title{
TAXONOMY OF LEISHMANIA. USE OF ISOENZYMES. SUGGESTIONS FOR A NEW CLASSIFICATION
}

\author{
J. A. RiouX, G. LANOTTE, E. SERRES, F. PRATlONG, \\ P. BASTIEN, J. PERIERES
}

\begin{abstract}
SUMMARY
The authors propose a new classification for the genus Leishmania Ross, 1903 based both on the use of intrinsic and extrinsic characters and on Linnean and Adansonian methods. The type of vertebrate host makes it possible to recognize the genus group: Leishmania designates Kinetoplastida parasites of mammals. Neighbouring forms which parasite Reptiles are now grouped in the genus Sauroleishmania Ranque, 1973. Characteristics of the intravectorial cycle (supra- and peri-pyloric) are used to define the subgenus group (Leishmania, Viannia Lainson and Shaw, 1987). The classification uses biochemical, particularly enzymatic, characters. Elementary taxonomic units are made up of all the strains having
\end{abstract}

the same isoenzyme profile, $i$.e. the zymodeme. The grouping of the zymodemes is usually performed through automatic techniques which lead to bush-like trees (dendrograms) showing either simple affinities between units (phenograms) or their phyletic relationships (cladograms). The branches recognized as being stable are individualized as "zymodeme complexes ». They bear the name of either the previously defined species taxa or that of a specially created one. Two examples of taxonomic constructions, phenetic and cladistic, are presented. Finally, a general classification of the genus is proposed.

\section{RÉsumé : Taxonomie des Leishmania. Utilisation des isoenzymes. Propositions pour une nouvelle classification.}

Les auteurs proposent une nouvelle classification du genre Leishmania Ross, 1903, basée sur l'utilisation de caractères intrinsèques et sur les méthodes linnéennes et adansoniennes. Le type d'hôte vertébré permet d'individualiser le groupe genre : les Leishmania désignent des Kinetoplastida parasites de Mammifères. Les formes voisines, intéressant les Reptiles, sont à présent regroupées dans le genre Sauroleishmania Ranque, 1973. Les caractéristiques du cycle intravectoriel (supra- et péripylorique) servent à définir le groupe sous-genre : Leishmania, Viannia Lainson et Shaw, 1987. En deçà, la classification utilise les caractères biochimiques, en particulier enzymatiques. Les unités taxonomiques élémentaires sont constituées par l'ensemble des souches présentant le même profil enzymatique, le zymodème. Le regroupement des zymodèmes s'effectue habituellement par les techniques automatiques. Il aboutit à des arbres buissonnants (dendrogrammes), traduisant soit les simples affinités entre unités (phénogramme), soit leurs relations phylétiques (cladogramme). Les rameaux reconnus comme stables sont individualisés en tant que "complexes de zymodèmes". Ils portent le nom des taxons spécifiques antérieurement définis ou créés à cette occasion. Deux exemples de construction taxonomique, phénétique et cladistique, sont présentés. In fine, une classification générale du genre est proposée.
Faced with a problem of systematics the parasitologist can adopt one of two attitudes, either that of the physician or epidemiologist for whom the identification of a pathogenic agent is an end in itself, or that of the naturalistecologist who attempts to reach biogeographic or phyletic levels of understanding.

The diagnostic approach is based on the search for discriminant characters which allow the individualization of a living object, $i$. e. its identification (« I. D. card»). This procedure can be extended to a group of objects. For organisms, such as Leishmania, which are difficult to separate by their morphological characters, the term used is

Laboratoire d'Écologie médicale, Faculté de Médecine, 163, rue Auguste-Broussonnet, F 34000 Montpellier.

Accepté le: 13 septembre 1990. typing. Typing generally involves intrinsic characters such as metabolic or genetic features. In some clinical forms of cutaneous leishmaniasis, typing of the causative organism should precede any prescription. The identification of L. braziliensis, now possible thanks to enzymatic methods, thus requires a prolonged treatment and both a careful clinical and immunological follow-up. In the same way, for cutaneous leishmaniasis around the Mediterranean basin, isolation of the viscerotropic zymodeme $L$. infantum MON-1 should suggest the possibility of spreading and lead ipso facto to a parenteral treatment with antimony. Ecoepidemiological analyses of foci of leishmaniasis demand the enzymatic identification of the parasite for the incrimination both of « true » vectors and of « true » reservoirs.

The classificatory approach seeks to define the relationships between objects or groups of objects. Like the diagnostic approach, it first focuses on analyzing the indivi- 
dual characters of elementary units. But it also goes beyond this stage to find those characters common to several group members. A return to base units, however, occurs when hierarchical constructions are used to elaborate dichotomous keys, which are in turn used for identification. It should finally be noted that most classifications include a notion of parental relationships (phylogeny).

\section{LINNEAN SYSTEMATICS OF THE GENUS LEISHMANIA. FROM SPECIES TO COMPLEXES AND FROM GENERA TO SUB-GENERA}

Since the creation of the genus Leishmania by Ross, 1903, the number of named species has continually increased. The descriptions of the binomials $L$. donovani Laveran and Mesnil, 1903, L. tropica Wright, 1903, L. infantum Nicolle, 1908, L. braziliensis Vianna, 1911, L. peruviana Velez, 1913, L. major Yakimoff and Schokhor, 1914, L. tarentolae Wenyon, 1921, L. enriettii Muniz and Medina, 1948, L. mexicana Biagi, 1953, L. guyanensis Floch, 1954 mark the first analytic period. Except for some taxa ( $L$. enriettii, L. major, L. mexicana) the criteria used for classification were extrinsic.

At the beginning of the $70 \mathrm{~s}$, a systematic regrouping of the taxa was carried out. It was formalized particularly by Lainson and Shaw's important studies on intravectorial cycles. The taxa were placed in three sections : Hypopylaria, Peripylaria and Suprapylaria $(23,24)$. Within these,

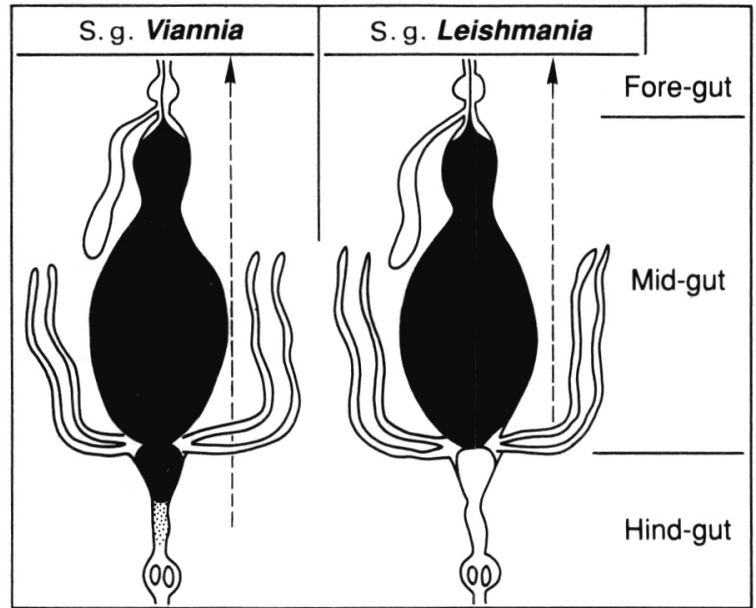

FIG. 1. - Sub-genus Leishmania Ross, 1903 and Viannia Lainson and Shaw, 1987. Classification based on behaviour in the gut of the sandlfy host (according to Lainson and Shaw 1979 and 1987).

and still based on extrinsic characters, the binomials were listed as sub-species ( $L$. donovani infantum, L. braziliensis guyanensis) which were in turn regrouped into complexes (L. donovani, L. braziliensis, etc.). In 1987, these same authors created the sub-genus Viannia to replace the Peripylaria section (25). The Suprapylaria section thus became a synonym for the sub-genus Leishmania (fig. 1). Prior to this, Reptile parasites, which had up to this time been placed in all three sections, Hypo-, Peri- and Suprapylaria,

TABlE I. - Reference strains of Leishmania species. Recommended by the Expert Commitee on Leishmaniasis of the World Health Organization (Geneva, 1989), these strains are deposited in the main international banks. Some are related to types (L. arabica, L. killicki), while others were designated at a later date by experts who considered the host and the country of the first description (43). Although they cannot be considered as neotypes, they nevertheless have their essential characters. Thus, the reference strain for L. infantum Nicolle, 1908 comes from a chid living near Tunis, suffering from visceral leishmaniasis. (CL = cutaneous leishmaniasis; $D C L=$ disseminated cutaneous; $M C L=$ muco-cutaneous; $V L=$ visceral).

L. aethiopica
L. amazonensis
L. arabica
L. archibaldi
L. aristedisi
L. braziliensis
L. chagasi
L. deanei
L. donovani
L. enriettii
L. garnhami
L. guyanensis
L. gerbilli
L. hertigi
L. infantum
L. killicki
L. lainsoni
L. major
L. mexicana
L. panamensis
L. peruviana
L. pifanoi
L. tropica
L. venezuelensis

L. aethiopica

amazonensis

L. archibaldi

L. aristedisi

L. chagasi

L. deanei

L. donovan

L. guyanensis

L. gerbilli

L. infantum

L. killicki

. lainsoni

L. mexicana

L. panamensis

. pifano

L. venezuelensis
MHOM/ET/72/L100

$\mathrm{MHOM} / \mathrm{BR} / 73 / \mathrm{M} 2269$

MPSA/SA/83/JISH220

Not selected

MORY/PA/69/GML3

MHOM/BR/00/LTB300

$\mathrm{MHOM} / \mathrm{BR} / 74 / \mathrm{M} 2682$

$\mathrm{MCOE} / \mathrm{BR} / 74 / \mathrm{M} 2674$

$\mathrm{MHOM} / \mathrm{IN} / 80 / \mathrm{DD} 8$

$\mathrm{MCAV} / \mathrm{BR} / 45 / \mathrm{L} 88$

$\mathrm{MHOM} / \mathrm{VE} / 76 / \mathrm{JAP} 78$

$\mathrm{MHOM} / \mathrm{BR} / 75 / \mathrm{M} 4147$

MRHO/CN/60/GERBILLI

$\mathrm{MCOE} / \mathrm{PA} / 65 / \mathrm{C} 8$

MHOM/TN/80/IPT1

MHOM/TN/80/LEM904

MHOM/BR/81/M6426

MHOM/SU/73/5-ASKH

$\mathrm{MHOM} / \mathrm{BZ} / 82 / \mathrm{BEL} 21$

MHOM/PA/71/LS94

Not selected

MHOM/VE/57/LL 1

$\mathrm{MHOM} / \mathrm{SU} / 74 / \mathrm{K} 27$

$\mathrm{MHOM} / \mathrm{VE} / 00 / \mathrm{H} 17$

\section{CL, DCL}

CL, DCL

Not observed in humans VL

Not observed in humans CL, MCL

CL, VL

Not observed in humans VL

Not observed in humans CL CL

Not observed in humans Not observed in humans CL, VL

CL

CL

CL

CL

CL

CL

DCL

CL

CL 
were gathered together into the genus Sauroleishmania Ranque, 1973 (19). Finally, following the recommendations of the Montpellier Symposium (Leishmania. Taxonomy and Phylogenesis, 1984) the trinominal nomenclature was abandoned and most of the taxa described at the beginning of the century were again given their species name (46).

It should be noted that the systematic categories thus defined involve a monothetic procedure and are based only on extrinsic criteria: clinical forms, epidemiological cycles (zoonosis, anthroponosis), host type, intravectorial behaviour, geographical distribution.

\section{BIOCHEMICAL ANALYSIS \\ AND ADANSONIAN SYSTEMATICS. \\ NUMERICAL TAXONOMY. \\ PHENETICS AND CLADISTICS}

\section{1 - PAST AND FUtURE}

In the $70 \mathrm{~s}$, the studies on biochemical (enzyme, DNA) and immunological (excreted factor) identification of Leishmania already focused on taxonomy $(8,11,12,53)$. They always referred to Linnean systematics, $i$. e. to the categories genus (Leishmania), sub-genus (Sauroleishmania), species and sub-species (L. infantum, L. tropica, L. braziliensis panamensis, etc.) (35).

From the beginning of the 80 s, attempts of automatic classification were made $(2,21,30,32,52)$. Initially they were simple hierarchical relationships (phenograms), although a certain reference to evolution could be seen in the chronological dendrograms (29). From this period on, it was also recommended to designate reference strains (standard, marker) and to protect them in cryobanks (table I).

Continuing the analytical effort, the next stage concentrated on evolution. It involved the application of Hennig's concepts and methods (17) and led to the construction of particular phyletic trees (cladograms). However, the absence of fossil remains made it necessary for the authors to reconsider the group origin (mono- or polyphyletic) and the status of characters (primitive or derived).

The next stage will probably involve molecular genetics. It will be based on the analysis of nuclear $(6,13)$ and kinetoplastic $(3,5)$ DNA and ribosomal RNA and will focus on the study of the fundamental mechanisms involved in speciation and taxonomic divergence. It will no doubt be long and difficult as the Trypanosomatidae, like many other Protozoa, represent primitive groups whose evolutionary strategies probably follow genetic rules quite different from those of modern Eukaryotes, particularly multicellular ones (59).

\section{2 - FROM ENZYMATIC CRITERIA}

TO CHEMOTAXONOMIC UNIT

As we have suggested above, difficulties can be found at two levels. The first one, risky and essential, is the cor- rect identification of the parasite and consequently the selection of the "right " characters; the second, more easily dealt with today, is the use of automatic classification techniques.

- Individualization of characters.

Electromorphs, isoenzymes and allozymes

In the classificatory model used the characters are represented by enzymatically active proteins called isoenzymes (isozymes). In fact, they represent alleles (alloenzymes, allozymes) generally coded at the same locus (genenzyme). In Leishmania, the genenzyme usually generates a single isoenzyme. This particular feature, which can be found in other asexually reproducing systematic groups, has always fascinated researchers. The rarity of heterozygous structures could be explained either by a typical haploidy, or by a diploidy with elimination of heterozygotes or repression of one of the alleles $(37,41,47)$.

In practice, the protein extracts are prepared from bulk cultures of promastigotes. The isoenzymes are separated in the form of electromorphs, after electrophoresis on starch gel (36), polyacrylamide gel (27) or cellulose acetate (20). Many difficulties are encountered at this stage with the techniques of migration and of electromorph revelation and identification. In general, it is preferable to use low voltages and extended migration times.

The selected enzymes must be both numerous and diversified. However, their number is often reduced due to difficulties during revelation and to reagent costs. Around 20 enzymes can be used if the selection effort is made 1) a priori, to retain the "stable» systems (structural enzymes, low sensibility to selection pressures), 2) a posteriori, to select non-redundant polymorphic systems, with, if possible, a phylogenetic relevance (plesiomorphs, synapomorphs). We currently use the following 15 enzymatic systems: malate dehydrogenase, $\mathrm{MDH}$ : EC 1.1.1.37; malic enzyme, ME : EC 1.1.1.40; isocitrate dehydrogenase, ICD : EC 1.1.1.42; phosphogluconate dehydrogenase, PGD : EC 1.1.1.44; glucose-6-phosphate dehydrogenase, G6PD : EC 1.1.1.49; glutamate dehydrogenase, GLUD : EC 1.4.1.3; diaphorase NADH, DIA : EC 1.6.2.2; nucleoside purine phosphorylase, NP1 : EC 2.4.2.1 and NP2 : EC 2.4.2.*; glutamate-oxaloacetate transaminase, GOT1 and GOT2 EC: 2.6.1.1, phosphoglucomutase, PGM : EC 2.7.5.1; mannose phosphate isomerase, MPI : EC 5.3.1.8; glucose phosphate isomerase, GPI : EC 5.3.1.9.

The electromorph can only be correctly identified by referring to an isoenzyme marker. This is a strain, or if possible a clone, selected for one or several electromorphs. These markers should be systematically included in the electrophoresis gels. Even more so than for the other strains, their biochemical integrity should be ensured by careful preservation. Currently, only liquid nitrogen fulfills the necessary conditions (cryobank). The protein extracts should 
TABLE II. - Enzyme profile of reference strains of Old World Leishmania.

\begin{tabular}{|c|c|c|c|c|c|c|c|c|c|c|c|c|c|c|c|c|c|}
\hline $\begin{array}{l}\text { Phenetic } \\
\text { complexes }\end{array}$ & $\begin{array}{c}\text { Zymodemes } \\
\text { MON- }\end{array}$ & $\mathrm{MDH}$ & ME & ICD & PGD & G6PD & GLUD & DIA & me pro & $\mathrm{NP}_{2}$ & $\mathrm{GOT}_{1}$ & $\mathrm{GOT}_{2}$ & PGM & $\mathrm{FH}$ & MPI & GPI & Reference strains \\
\hline \multirow{18}{*}{ L. infantum } & 1 & 100 & 100 & 100 & 100 & 100 & 100 & 100 & 100 & 100 & 100 & 100 & 100 & 100 & 100 & 100 & MHOM/FR/78/LEM 75 \\
\hline & 11 & 104 & 100 & 100 & 100 & 105 & 100 & 100 & 130 & 100 & 100 & 100 & 100 & 100 & 100 & 100 & MHOM/FR/80/LEM 189 \\
\hline & 24 & 104 & 100 & 100 & 100 & 100 & 100 & 100 & 140 & 100 & 100 & 100 & 100 & 100 & 100 & 100 & MHOM/DZ/82/LIPA 59 \\
\hline & 27 & 100 & 100 & 100 & 100 & 100 & 100 & 100 & 130 & 100 & 100 & 100 & 100 & 100 & 100 & 100 & MHOM/IT/79/ISS 7 \\
\hline & 28 & 104 & 100 & 100 & 100 & 102 & 100 & 100 & 140 & 100 & 100 & 100 & 100 & 100 & 100 & 100 & MHOM/ES/83/BCN 2 \\
\hline & 29 & 104 & 100 & 100 & 100 & 105 & 100 & 100 & 140 & 100 & 100 & 100 & 100 & 100 & 100 & 100 & MHOM/ES/81/BCN 1 \\
\hline & 30 & 112 & 100 & 100 & 100 & 100 & 100 & 100 & 140 & 100 & 100 & 100 & 100 & 100 & 100 & 100 & MHOM/SD/82/GILAN! \\
\hline & 33 & 104 & 100 & 100 & 100 & 105 & 100 & 100 & 100 & 100 & 100 & 100 & 100 & 100 & 100 & 100 & MHOM/FR/82/LEM 356 \\
\hline & 34 & 104 & 100 & 100 & 100 & 100 & 100 & 100 & 100 & 100 & 100 & 100 & 100 & 100 & 100 & 100 & MHOM/FR/84/LEM 538 \\
\hline & 72 & 100 & 100 & 100 & 100 & 100 & 100 & 100 & 100 & 100 & 100 & 100 & 109 & 100 & 100 & 100 & MHOM/IT/86/ISS 218 \\
\hline & 77 & 100 & 100 & 100 & 100 & 102 & 100 & 100 & 100 & 100 & 100 & 100 & 100 & 100 & 100 & 100 & MCAN/ES/86/LEM 935 \\
\hline & 78 & 104 & 100 & 100 & 100 & 100 & 100 & 100 & 140 & 100 & 100 & 100 & 100 & 110 & 100 & 100 & MHOM/MT/85/BUCK \\
\hline & 80 & 104 & 100 & 100 & 100 & 100 & 100 & 100 & 130 & 100 & 100 & 100 & 100 & 100 & 100 & 100 & MHOM/DZ/83/LEM 425 \\
\hline & 81 & 112 & 100 & 100 & 100 & 100 & 100 & 100 & 150 & 100 & 100 & 100 & 100 & 100 & 100 & 100 & MHOM/SD/00/3 S \\
\hline & 98 & 100 & 90 & 100 & 100 & 100 & 100 & 100 & 100 & 100 & 100 & 100 & 100 & 100 & 100 & 100 & MHOM/EG/87/RTC 2 \\
\hline & 105 & 100 & 100 & 100 & 100 & 105 & 100 & 100 & 100 & 100 & 100 & 100 & 100 & 100 & 100 & 100 & MCAN/ES/88/LEM 1355 \\
\hline & 108 & 100 & 100 & 100 & 100 & 100 & 100 & 120 & 100 & 100 & 100 & 100 & 100 & 100 & 100 & 100 & MCAN/FR/87/RM 1 \\
\hline & 111 & 104 & 100 & 100 & 100 & 100 & 100 & 100 & 140 & 100 & 100 & 100 & 100 & 100 & 120 & 100 & MHOM/IT/85/ISS 175 \\
\hline
\end{tabular}

\begin{tabular}{|c|c|c|c|c|c|c|c|c|c|c|c|c|c|c|c|c|c|}
\hline \multirow{13}{*}{ L. donovani } & 2 & 104 & 100 & 100 & 93 & 100 & 100 & 100 & 140 & 100 & 113 & 113 & 100 & 100 & 100 & 100 & MHOM/IN/OO/DEV I \\
\hline & 3 & 104 & 100 & 100 & 100 & 100 & 100 & 100 & 140 & 100 & 113 & 113 & 100 & 100 & 100 & 86 & $\mathrm{MHOM} / \mathrm{IQ} / 73 / \mathrm{IF} 3$ \\
\hline & 18 & 112 & 100 & 100 & 100 & 100 & 100 & 100 & 140 & 100 & 113 & 113 & 100 & 100 & 100 & 100 & MHOM/ET/67/HU 3 \\
\hline & 31 & 104 & 100 & 100 & 100 & 100 & 100 & 100 & 140 & 100 & 113 & 113 & 100 & 100 & 110 & 86 & MHOM/SA/81/JEDDAH-KA \\
\hline & 32 & 104 & 100 & 100 & 100 & 100 & 100 & 100 & 150 & 100 & 113 & 113 & 100 & 100 & 100 & 100 & MHOM/KE/75/H 9 \\
\hline & 35 & 104 & 100 & 100 & 100 & 100 & 150 & 100 & 150 & 100 & 113 & 113 & 100 & 100 & 100 & 86 & MHOM/CN/0O/WANGJIE-1 \\
\hline & 36 & 104 & 100 & 100 & 100 & 100 & 100 & 100 & 100 & 100 & 113 & 113 & 100 & 100 & 100 & 100 & MHOM/KE/55/LRC-L 53 \\
\hline & 37 & 104 & 100 & 100 & 100 & 100 & 100 & 100 & 140 & 100 & 113 & 113 & 100 & 100 & 100 & 100 & IMAR/KE/62/LRC-L 57 \\
\hline & 38 & 100 & 100 & 100 & 100 & 100 & 100 & 100 & 100 & 100 & 113 & 113 & 100 & 100 & 100 & 100 & MHOM/IN/54/SC 23 \\
\hline & 73 & 104 & 100 & 100 & 100 & 100 & 100 & 100 & 140 & 100 & 113 & 113 & 100 & 110 & 100 & 100 & MHOM/SU/84/MARZ-KRIM \\
\hline & $82^{*}$ & 112 & 100 & 100 & 100 & 100 & 100 & 100 & 140 & 100 & 110 & 110 & 100 & 100 & 100 & 100 & MHOM/ET/72/GEBRE 1 \\
\hline & 83 & 104 & 100 & 100 & 100 & 100 & 100 & 100 & 140 & 100 & 113 & 113 & 100 & 98 & 110 & 86 & MHOM/ET/84/ADDIS 164 \\
\hline & 84 & 112 & 100 & 100 & 100 & 100 & 100 & 100 & 150 & 100 & 113 & 113 & 100 & 100 & 100 & 100 & MHOM/MT/00/MALTA 33 \\
\hline
\end{tabular}

* L. archibaldi 
TABLE II. - (following).

\begin{tabular}{|c|c|c|c|c|c|c|c|c|c|c|c|c|c|c|c|c|c|}
\hline $\begin{array}{l}\text { Phenetic } \\
\text { complexes }\end{array}$ & $\begin{array}{l}\text { Zymodemes } \\
\text { MON- }\end{array}$ & $\mathrm{MDH}$ & ME & ICD & PGD & G6PD & GLUD & $\begin{array}{l}\text { Enzy } \\
\text { DIA }\end{array}$ & $\begin{array}{l}\text { me pr } \\
\begin{array}{l}N P_{1}\end{array}\end{array}$ & $N P_{2}$ & GOT & $\mathrm{GOT}_{2}$ & PGM & $\mathrm{FH}$ & $\mathrm{MPI}$ & GPI & Reference strains \\
\hline \multirow{28}{*}{ L. tropica } & 5 & 112 & 95 & 100 & 93 & 82 & 80 & 100 & 450 & 100 & 135 & 90 & 100 & 110 & 110 & 76 & MRAT/IQ/72/ADHANIS 1 \\
\hline & 6 & 100 & 95 & 100 & 93 & 82 & 95 & 110 & 450 & 100 & 135 & 90 & 100 & 100 & 101 & 76 & MHOM/IQ/65/BRAY L 75 \\
\hline & 7 & 112 & 95 & 100 & 93 & 82 & 95 & 110 & 450 & 100 & 135 & 90 & 100 & 100 & 101 & 76 & MHOM/PK/00/LV 691 \\
\hline & $8^{*}$ & 100 & 100 & 100 & 93 & 82 & 110 & 100 & 300 & 100 & 127 & 90 & 100 & 100 & 110 & 76 & MHOM/TN/80/LEM 163 \\
\hline & 13 & 112 & 95 & 100 & 93 & 82 & 95 & 100 & 450 & 100 & 135 & 90 & 100 & 110 & 101 & 76 & MHOM/LB/76/LEM 61 \\
\hline & 19 & 100 & 90 & 100 & 105 & 82 & 80 & 100 & 450 & 110 & 140 & 85 & 90 & 100 & 110 & 60 & MHOM/NA/74/BRANDT \\
\hline & 20 & 100 & 100 & 100 & 105 & 82 & 80 & 100 & 450 & 110 & 140 & 85 & 90 & 100 & 110 & 60 & IROS/NA/80/ROSSI 80 \\
\hline & 39 & 100 & 95 & 100 & 95 & 82 & 95 & 100 & 450 & 90 & 135 & 90 & 100 & 100 & 101 & 76 & MHOM/IQ/65/AV SINAI \\
\hline & 53 & 112 & 95 & 100 & 95 & 82 & 95 & 110 & 450 & 90 & 135 & 90 & 100 & 100 & 101 & 76 & MHOM/IR/70/NADIM 3 \\
\hline & 54 & 112 & 95 & 100 & 100 & 82 & 80 & 120 & 450 & 90 & 135 & 90 & 100 & 110 & 100 & 76 & MHOM/IL/80/SINGER \\
\hline & 55 & 112 & 95 & 100 & 95 & 82 & 95 & 100 & 450 & 90 & 135 & 90 & 100 & 100 & 101 & 76 & $\mathrm{MHOM} / \mathrm{TR} / 83 / \mathrm{ISS} 68$ \\
\hline & 56 & 118 & 95 & 100 & 93 & 82 & 80 & 120 & 340 & 90 & 135 & 90 & 100 & 110 & 100 & 76 & MHOM/GR/80/GR-L 35 \\
\hline & 57 & 118 & 100 & 100 & 95 & 82 & 80 & 120 & 450 & 90 & 135 & 90 & 100 & 110 & 110 & 76 & MHOM/GR/82/SER-L 60 \\
\hline & 58 & 112 & 95 & 100 & 93 & 82 & 95 & 100 & 450 & 100 & 135 & 90 & 108 & 100 & 101 & 76 & $\mathrm{MHOM} / \mathrm{AF} / 82 / \mathrm{K} 001$ \\
\hline & 59 & 112 & 100 & 100 & 93 & 82 & 95 & 100 & 450 & 100 & 135 & 90 & 100 & 100 & 100 & 76 & MHOM/AF/82/K 006 \\
\hline & 60 & 100 & 95 & 100 & 93 & 82 & 95 & 100 & 450 & 100 & 135 & 90 & 108 & 100 & 101 & 76 & MHOM/SU/74/K 27 \\
\hline & 61 & 112 & 95 & 100 & 95 & 82 & 95 & 100 & 450 & 100 & 135 & 90 & 108 & 100 & 101 & 76 & $\mathrm{MHOM} / \mathrm{SU} / 66 \mathrm{~N}$ \\
\hline & 62 & 112 & 95 & 100 & 93 & 82 & 95 & 110 & 450 & 90 & 135 & 90 & 100 & 110 & 101 & 76 & MCAN/IN/71/DBKM \\
\hline & 63 & 112 & 95 & 100 & 93 & 82 & 80 & 110 & 450 & 100 & 135 & 90 & 100 & 100 & 101 & 76 & MHOM/ET/00/LV 471 \\
\hline & 71 & 100 & 100 & 100 & 93 & 82 & 80 & 100 & 450 & 110 & 140 & 85 & 100 & 98 & 110 & 76 & MHOM/YE/86/LEM 955 \\
\hline & 75 & 112 & 95 & 100 & 95 & 82 & 95 & 100 & 450 & 100 & 135 & 90 & 100 & 100 & 101 & 76 & MHOM/SU/58/OD \\
\hline & 76 & 112 & 95 & 100 & 93 & 82 & 95 & 110 & 450 & 100 & 135 & 90 & 108 & 110 & 101 & 76 & MHOM/SY/86/LIPA 154 \\
\hline & 102 & 118 & 95 & 100 & 95 & 82 & 80 & 120 & 450 & 90 & 130 & 70 & 108 & 110 & 100 & 76 & MHOM/MA/88/LEM 1314 \\
\hline & 104 & 100 & 95 & 100 & 95 & 82 & 95 & 110 & 450 & 100 & 135 & 90 & 100 & 100 & 101 & 76 & 0000/00/84/LEM 643 \\
\hline & 107 & 104 & 80 & 100 & 95 & 85 & 80 & 120 & 420 & 110 & 135 & 90 & 109 & 110 & 110 & 88 & MHOM/MA/88/LEM 1452 \\
\hline & 109 & 118 & 95 & 100 & 108 & 82 & 80 & 120 & 450 & 90 & 130 & 70 & 108 & 110 & 100 & 76 & MHOM/MA/89/LEM 1591 \\
\hline & 112 & 118 & 95 & 100 & 102 & 82 & 80 & 120 & 450 & 90 & 130 & 70 & 108 & 110 & 100 & 76 & MHOM/MA/88/LEM 1451 \\
\hline & 113 & 112 & 95 & 100 & 95 & 82 & 95 & 100 & 450 & 100 & 128 & 90 & 108 & 100 & 101 & 76 & MHOM/MA/89/LEM 1777 \\
\hline
\end{tabular}

\begin{tabular}{|l|l|l|l|l|l|l|l|l|l|l|l|l|l|l|l|l|l|}
\hline & 14 & 130 & 108 & 100 & 140 & 82 & 100 & 100 & 700 & 120 & 127 & 110 & 123 & 105 & 105 & 53 & MHOM/ET/72/L 100 \\
\cline { 2 - 14 } \\
\cline { 2 - 11 }
\end{tabular}

* L. killicki 
TABle II. - (end).

\begin{tabular}{|c|c|c|c|c|c|c|c|c|c|c|c|c|c|c|c|c|c|}
\hline $\begin{array}{l}\text { Phenetic } \\
\text { complexes }\end{array}$ & $\begin{array}{c}\text { Zymodemes } \\
\text { MON- }\end{array}$ & \multicolumn{15}{|c|}{ Enzyme profiles } & Reference strains \\
\hline \multirow{12}{*}{ L. major } & 4 & 160 & 88 & 90 & 122 & 94 & 200 & 100 & 400 & 90 & 110 & 110 & 118 & 79 & 150 & 77 & MRH0/SU/59/P-strain \\
\hline & 21 & 160 & 108 & 130 & 140 & 94 & 220 & 100 & 500 & 110 & 110 & 110 & 118 & 70 & 110 & 77 & $\mathrm{MRHO} / \mathrm{SU} / 65 \mathrm{NL}$ \\
\hline & 23 & 160 & 88 & 90 & 122 & 94 & 200 & 100 & 400 & 100 & 110 & 110 & 118 & 79 & 150 & 65 & MMER/IN/73/GTBM \\
\hline & 25 & 150 & 88 & 100 & 122 & 94 & 200 & 100 & 400 & 90 & 110 & 120 & 118 & 87 & 150 & 77 & MHOM/MA/81/LEM 265 \\
\hline & 26 & 160 & 88 & 100 & 122 & 94 & 200 & 100 & 400 & 90 & 110 & 110 & 118 & 79 & 150 & 77 & MHOM/YD/76/LEM 62 \\
\hline & 64 & 160 & 100 & 130 & 150 & 94 & 220 & 100 & 500 & 120 & 110 & 110 & 118 & 70 & 150 & 77 & $\mathrm{MRHO} / \mathrm{SU} / 74 / 95 \mathrm{~A}$ \\
\hline & 65 & 160 & 108 & 130 & 140 & 94 & 220 & 100 & 500 & 120 & 110 & 110 & 118 & 70 & 150 & 77 & MMEL/SU/79/Mel \\
\hline & 66 & 160 & 88 & 100 & 130 & 94 & 200 & 100 & 400 & 90 & 110 & 110 & 110 & 79 & 150 & 77 & MHOM/IL/83/IL 24 \\
\hline & 67 & 160 & 88 & 100 & 130 & 94 & 200 & 100 & 400 & 90 & 110 & 110 & 118 & 79 & 150 & 77 & MHOM/IL/83/IL 53 \\
\hline & 68 & 160 & 88 & 100 & 122 & 94 & 200 & 100 & 400 & 110 & 110 & 110 & 110 & 79 & 150 & 77 & MHOM/IL/83/IL 32 \\
\hline & 74 & 160 & 88 & 100 & 122 & 94 & 200 & 90 & 400 & 90 & 110 & 110 & 118 & 79 & 150 & 77 & MCAN/EG/OO/ASC-D 1 \\
\hline & 103 & 160 & 88 & 100 & 122 & 94 & 200 & 100 & 400 & 100 & 110 & 110 & 118 & 79 & 150 & 77 & MPSA/J0/87/JPs-G \\
\hline
\end{tabular}

\begin{tabular}{|l|c|c|c|c|c|c|c|c|c|c|c|c|c|c|c|c|c|}
\hline L. gerbilli & 22 & 104 & 95 & 120 & 130 & 94 & 250 & 100 & 350 & 120 & 150 & 80 & 118 & 79 & 130 & 55 & MRHO/CN/60/Gerbilli \\
\hline
\end{tabular}

\begin{tabular}{|l|l|l|l|l|l|l|l|l|l|l|l|l|l|l|l|l|l|}
\hline L. arabica & 99 & 150 & 93 & 100 & 140 & 87 & 220 & 100 & 350 & 100 & 120 & 30 & 118 & 60 & 160 & 50 & MPSA SA $83 /$ JISH 220 \\
\hline
\end{tabular}

TABLE III. - Enzyme profile of reference strains of the New World Leishmania.

\begin{tabular}{|c|c|c|c|c|c|c|c|c|c|c|c|c|c|c|c|}
\hline Phenetic complexes & $\begin{array}{l}\text { Zymodemes } \\
\text { MON- }\end{array}$ & ME & $I C D$ & PGD & G6PD & $D I A$ & $\begin{array}{l}\text { Enzy } \\
N P_{1}\end{array}$ & $\begin{array}{l}\text { me pro } \\
N_{2}\end{array}$ & $\begin{array}{l}\text { ofiles } \\
\text { GOT }_{1}\end{array}$ & $\mathrm{GOT}_{2}$ & PGM & $\mathrm{FH}$ & MPI & GPI & Reference strains \\
\hline \multirow{2}{*}{ L. braziliensis } & 43 & $A^{3}$ & $B^{1}$ & $c^{4}$ & $D^{4}$ & $E^{2}$ & $F^{3}$ & $G^{4}$ & $H^{4}$ & 15 & $J^{2}$ & $K^{2}$ & $L^{2}$ & $M^{4}$ & MHOM/BR/75/M 2903 \\
\hline & 44 & $A^{2}$ & $B^{1}$ & $C^{4}$ & $D^{4}$ & $E^{2}$ & $\mathrm{~F}^{3}$ & $\mathrm{G}^{4}$ & $\mathrm{H}^{4}$ & $1^{5}$ & $J^{2}$ & $\mathrm{~K}^{2}$ & $L^{2}$ & $M^{4}$ & MHOM/CO/83/LEM 469 \\
\hline
\end{tabular}

\begin{tabular}{|l|l|l|l|l|l|l|l|l|l|l|l|l|l|l|l|}
\hline \multirow{5}{*}{ L. guyanensis } & 47 & $\mathrm{~A}^{2}$ & $\mathrm{~B}^{2}$ & $\mathrm{C}^{2}$ & $\mathrm{D}^{5}$ & $\mathrm{E}^{3}$ & $\mathrm{~F}^{1}$ & $\mathrm{G}^{3}$ & $\mathrm{H}^{5}$ & $\mathrm{I}^{3}$ & $\mathrm{~J}^{1}$ & $\mathrm{~K}^{3}$ & $\mathrm{~L}^{1}$ & $\mathrm{M}^{4}$ & $\mathrm{MHOM} / \mathrm{PA} / 75 / \mathrm{M} 4037$ \\
\cline { 2 - 13 } & 51 & $\mathrm{~A}^{2}$ & $\mathrm{~B}^{2}$ & $\mathrm{C}^{3}$ & $\mathrm{D}^{6}$ & $\mathrm{E}^{3}$ & $\mathrm{~F}^{1}$ & $\mathrm{G}^{3}$ & $\mathrm{H}^{5}$ & $\mathrm{I}^{3}$ & $\mathrm{~J}^{1}$ & $\mathrm{~K}^{3}$ & $\mathrm{~L}^{1}$ & $\mathrm{M}^{4}$ & $\mathrm{MHOM} / \mathrm{CO} / 83 / \mathrm{REST} 417$ \\
\cline { 2 - 13 } & 100 & $\mathrm{~A}^{2}$ & $\mathrm{~B}^{2}$ & $\mathrm{C}^{2}$ & $\mathrm{D}^{6}$ & $\mathrm{E}^{3}$ & $\mathrm{~F}^{1}$ & $\mathrm{G}^{3}$ & $\mathrm{H}^{5}$ & $\mathrm{I}^{3}$ & $\mathrm{~J}^{1}$ & $\mathrm{~K}^{3}$ & $\mathrm{~L}^{1}$ & $\mathrm{M}^{4}$ & $\mathrm{MHOM} / \mathrm{CO} / 83 / 487$ \\
\cline { 2 - 12 } & 101 & $\mathrm{~A}^{2}$ & $\mathrm{~B}^{2}$ & $\mathrm{C}^{1}$ & $\mathrm{D}^{6}$ & $\mathrm{E}^{3}$ & $\mathrm{~F}^{1}$ & $\mathrm{G}^{3}$ & $\mathrm{H}^{5}$ & $\mathrm{I}^{3}$ & $\mathrm{~J}^{1}$ & $\mathrm{~K}^{3}$ & $\mathrm{~L}^{1}$ & $\mathrm{M}^{4}$ & $\mathrm{MHOM} / \mathrm{CO} / 86 / \mathrm{UA} 126$ \\
\hline
\end{tabular}

\begin{tabular}{|l|l|l|l|l|l|l|l|l|l|l|l|l|l|l|l|}
\hline \multirow{3}{*}{ L. mexicana } & 40 & $\mathrm{~A}^{4}$ & $\mathrm{~B}^{3}$ & $C^{7}$ & $D^{2}$ & $E^{4}$ & $F^{2}$ & $\mathrm{G}^{2}$ & $H^{1}$ & $\mathrm{I}^{2}$ & $J^{4}$ & $K^{4}$ & $\mathrm{~L}^{4}$ & $M^{1}$ & $\mathrm{MNYC} / \mathrm{BZ} / 62 / \mathrm{M} 379$ \\
\cline { 2 - 11 } & 41 & $\mathrm{~A}^{4}$ & $\mathrm{~B}^{5}$ & $\mathrm{C}^{7}$ & $\mathrm{D}^{2}$ & $\mathrm{E}^{5}$ & $F^{1}$ & $\mathrm{G}^{2}$ & $\mathrm{H}^{2}$ & $\mathrm{I}^{2}$ & $J^{1}$ & $\mathrm{~K}^{4}$ & $\mathrm{~L}^{4}$ & $M^{2}$ & $\mathrm{IFLA} / \mathrm{BR} / 67 / \mathrm{PH} 8$ \\
\hline
\end{tabular}

\begin{tabular}{|l|l|l|l|l|l|l|l|l|l|l|l|l|l|l|l|}
\hline L. enriettii & 97 & $A^{1}$ & $B^{4}$ & $C^{7}$ & $D^{3}$ & $E^{1}$ & $F^{1}$ & $G^{5}$ & $H^{3}$ & $I^{4}$ & $J^{1}$ & $K^{1}$ & $L^{5}$ & $M^{3}$ & $M C A V / B R / 45 / L 88$ \\
\hline
\end{tabular}

\begin{tabular}{|l|l|l|l|l|l|l|l|l|l|l|l|l|l|l|l|}
\hline L. hertigi & 52 & $A^{5}$ & $\mathrm{~B}^{3}$ & $C^{6}$ & $D^{1}$ & $\mathrm{E}^{2}$ & $F^{4}$ & $\mathrm{G}^{1}$ & $\mathrm{H}^{4}$ & $\mathrm{I}^{1}$ & $J^{3}$ & $\mathrm{~K}^{4}$ & $\mathrm{~L}^{3}$ & $\mathrm{M}^{2}$ & $\mathrm{MCOE} / \mathrm{BR} / 78 / \mathrm{M} 5088$ \\
\hline
\end{tabular}


nevertheless be used rapidly ( $<12$ months). The recognition of a new electromorph needs special care, particularly for slight differences in migration distances: it is often necessary to repeat the migrations to confirm the authenticity of the electromorph in question. At this stage, modifications of $p \mathrm{H}$, voltage and migration time can be useful.

Any identification of a strain should be based on the complete list of its electromorphs (e. g. G6PD 100, $\mathrm{MDH} 112$, etc.). Along these lines a relevant synthesis of notation and designation modes for enzymes and their isoenzymes was published by Chance (7). This author particularly recommends using a standard reference strain which allows the calculation of the mobility of the different enzymes according to the formula: relative mobility of the $i$ band $=$ (distance from the start of the $i$ band $/$ distance from the start of the reference band) $\times 100$. For the past several years, this recommendation has been rigorously followed by the Montpellier Center. The standard strain's international code is MHOM/FR/78/LEM75. The corresponding extracts must be continually available (continuous bulk culture). It should be noted that no enzymatic changes in this strain have been found since its isolation (10 years in cryopreservation along with continuous culture).

\section{- Definition of the taxonomic unit, the zymodeme}

The enzymatic characters thus identified allow the individualization of the zymodeme, a concept proposed by Godfrey (14). By definition, the zymodeme corresponds to all the strains with the same enzymatic profile. The zymodeme thus constitutes a taxon whose systematic level is undefined, $i$. $e$. one that does no refer to a "category" recognized by the Nomenclature Code. It is an elementary phenon as defined by Sokal and Sneath (57). This position has been adopted by most researchers since, at the available evidence, the mode of evolution in Leishmania is still considered as clonal. Then the zymodeme would represent the true evolutionary unit. In the light of recent findings, it appears nevertheless possible that sexual (or parasexual) phenomena occur (see below). If these phenomena were essential, some groups of finely differentiated zymodemes (« small variants ») would simply represent the " populational expression » of the same biological entity (38). However, the present evidence does not allow to draw such conclusions.

The choice of the zymodeme as a unit raises another difficulty: the use of additional enzymes may lead to the break up of the zymodeme into two or several new units. Consequently, for each zymodeme, it is essential to designate one strain (clone if possible) as a reference which will remain attached to it (tables II and III). This clone then will allow, at least partially, the recognition of synonymies by the Centers of enzymatic identification which often use non totally overlapping techniques. In order to avoid any confusion, each Center should also preferably use an independent logo (LON-for London, MON-for Montpellier) (50).

\section{3 - From Adansonian concepts} TO AUTOMATIC CLASSIFICATIONS

The essential notions of numerical taxonomy were anticipated two centuries ago by the French botanist Adanson (1). He imagined the concomitant use of numerous characters (polythetism), rather can the successive use of some of them (monothetism). The development of computers has made it possible to put Adanson's concepts in practice. Thanks to North-American and British researchers, this method has today reaped its just reward (56).

It should be recalled that this type of taxonomy requires a «large number » of characters (variables, descriptors, « signifiers ») and a « large number » of units (objects, phenons, "signified »). The numerical classification of Leishmania is no exception. The characters, called unitary, are represented by isoenzymes; the operational taxonomic units (OTUs) are represented by the zymodemes. The affinities, or resemblances, between units are calculated using distance coefficients chosen according to the objective of the study (Jaccard index, Nei index, Manhattan index, Euclidian distance, etc.). After coding the characters, several classificatory techniques can be used. The agglomerative hierarchical methods are best adapted to enzymatic data analysis (54). To perform this, several algorithms are available using simple linkage, group average linkage or complete linkage $(34,51)$. The result is a bush-like tree diagram (phenetic dendrogram or phenogram) which expresses the affinities between OTUs and OTU groups. The authenticity in terms of objective divisions $(9,54)$ of the branches obtained can be verified by other procedures, such as the moving center analysis or ordination techniques (multidimensional analysis).

- Phenetics, chronistics and cladistics

Numerical taxonomy leading to the development of tree diagrams fulfills three objectives:

1 - Simple hierarchical classification, $i$. e. without reference to time or to evolution $=$ phenetic tree. The characters are given the same weight and no hypothesis is formulated as to the independence of the sub-groups identified by the dendrograms (fig. 2 and 4).

2 - Calculation of divergence time between phenons or phenon groups $=$ chronistic tree. This method considers the genetic significance of the characters. The new distance, called genetic distance, expresses the number of replacements of internal nucleotides at each site (mutation). In every case, it presupposes genenzyme neutrality and constant mutation rate. Nei, the promoter of this method, has generalized it to asexual organisms (42). 


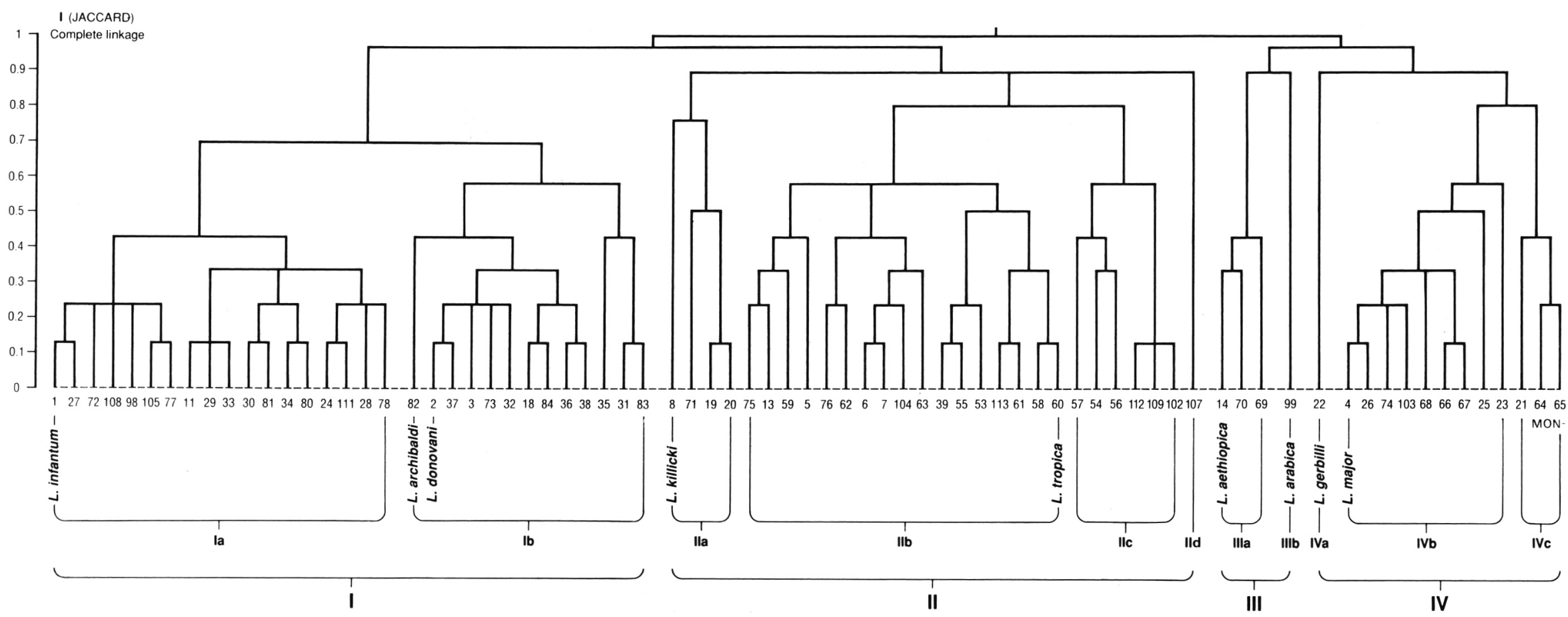

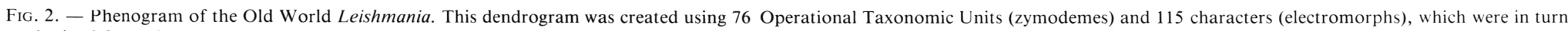
obtained from the enzymatic analysis of 752 strains. Several similar constructions are possible. In all cases, four main groups, L. donovani s. I. (I), L. tropica s. 1. (II), L. aethiopica (III) and $L$, major (IV) are present. Within group I, $L$. infantum (Ia) is always separated from $L$ donovani s. s. (Ib). But $L$ archibaldi (MON-82) can either be in I $a$ or in Ib. Within the group II, cluster II $a$ formed by four zymodemes, MON-8, i. e. L. killicki (49), MON-71 from Yemen, and MON-19 and MON-20 from Namibia, and cluster II $b$ formed by 17 zymodemes, are clearly distinct. Other zymodemes originating from proximal western Asia (MON-54, MON-56, MON-57) and from Morocco (MON-102, MON-109, MON-112) have a clear tendency to regrouping (IIc), but in some phenograms they cannot be separated from sub-group IIb. Still within the $L$. tropica group, the sub-group IId, represented by zymodeme MON-107, does not take a definite position when several phenograms are compared. Within group III, $L$. arabica (III $b)$ is distinct from the closely related zymodemes of $L$. aethiopica s. l. (III a), whatever phenogram is considered. Within group IV, three sub-groups are stable: $L$. gerbilli (IV $a$ ), clearly separated from the two others, one sub-group enclosing the $L$. major s. s. zymodemes (IVb), and the cluster formed by MON-21, MON-64 and MON-65 (IVc), three zymodemes from USSR.

Within each of the stable sub-groups, i. e. the sub-groups always formed by the same unities, the relative positions of zymodemes may nevertheless vary from one phenogram to another. This « unstability » reflects the existence of an identical number of links between several zymodemes giving them the same index value (" ex-aequo »). 
3 - Phyletic analysis emphasized since Hennig. This author's considerations made many fruitful contacts possible between evolutionists and pheneticians. A new science was born, cladistics, which allows the elaboration of « evolutionary dendrograms " (cladograms). Cladistics thus definitely advanced the phyletic understanding of many groups (15). Concepts such as monophyletism, parsimony, succession and non-convergence of the characters are largely responsible for its spread. In the genus Leishmania, this type of construction is always based on the zymodeme, $i$. e. the OTU used in phenetics (fig. 3 and 5). However, the characters here are genenzymes and not allozymes. The latter are reduced to simple " character states » (4). A presumed ancestor, the hypothetical taxonomic unit (HTU), is placed at each branching. The lowest HTU corresponds to the common ancestor of the studied set of OTUs. In Leishmania, as in most parasitic Protozoa, the absence of fossil remains, of a living ancestor or of a recognized

L. tropica compl.

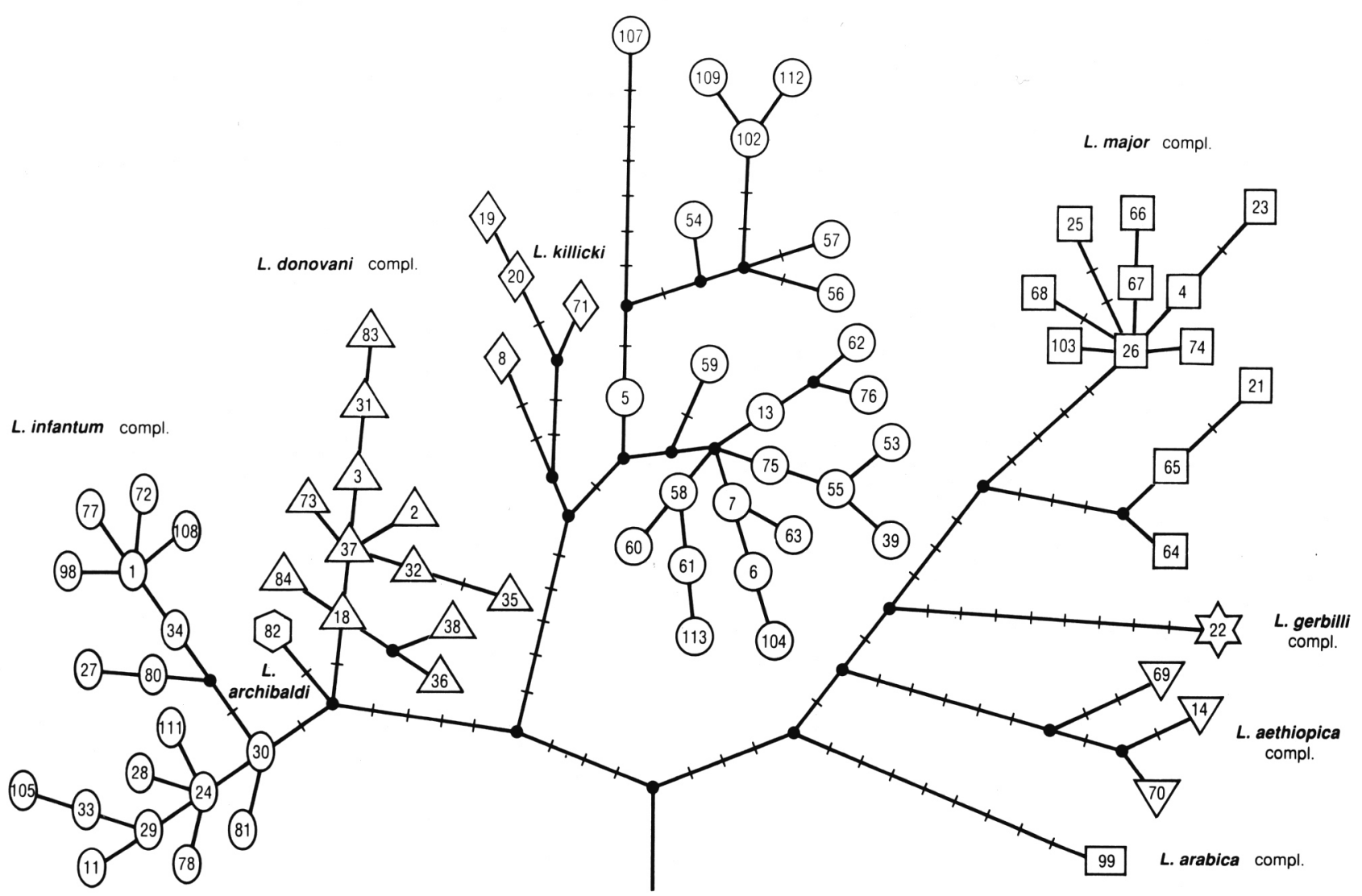

FIG. 3. - Cladogram of Old World Leishmania. This was constructed using 15 characters (genenzymes) and 115 states (allozymes) for 76 Operational Taxonomic Units (zymodemes). The present tree is the most parsimonious found. The groups appearing are similar to those on the phenogram. Nevertheless, as it takes into account not only the number but also the intrinsic value of the common characters states, it allows certain details to be clarified, in particular the separation, just-above the root, of two evolutionary groups: one including L. infantum, L. donovani and L. tropica, the other L. arabica, L. aethiopica, L. gerbilli and L. major. Moreover, the cladogram vizualises the distances between the different OTUs. Hence, following groups are better individualized: 1) $L$. arabica; 2 ) $L$. gerbilli; 3) the USSR sub-group (MON-21, MON-64, MON-65), up to now included in the L. major s. s. group; 4) the cluster formed with zymodemes from western Asia and Morocco (MON-54, MON-56, MON-57, MON-102, MON-107, MON-109, MON-112), within the L. tropica group; 5) the L. killicki cluster (MON-8, MON-19, MON-20, MON-71). It should be noted that this cluster is ancestral in relation to the other zymodemes of $L$. tropica and that the Namibian zymodeme MON-19 affects Hyracoïds as well as Man. This observation argues in favor of a zoonotic origin for the L. tropica complex.

The cladogram brings forward other precisions. Thus, MON-107 is located near the Moroccan group (MON-102, MON-109, MON-112), originating from the same geographical area. MON-82 is distinct from the L. infantum and L. donovani " bushes", giving rise to a trifurcated structure. The structure thus formed by L. infantum, L. archibaldi (MON-82) and L. donovani, is directly dependent on the glutamate-oxaloacetate transaminases (GOT1 and GOT2) variation. It should be emphasized that MON-82 (L. archibaldi), as well as the ancestral zymodeme (MON-18) of the L. donovani group and the ancestral zymodeme (MON-30) of the $L$. infantum group, are originating from Sudan.

Lastly, the cladogram shows better than the phenogram the ambiguity of the relations between zymodemes, within most of the groups: numerous zymodemes as ancestors, numerous zymodemes issued from the same ancestor (« rake» or «bunch» pattern). 
« sister group» makes it impossible to identify the root of the tree, $i . e$. to orientate it. It is then necessary to

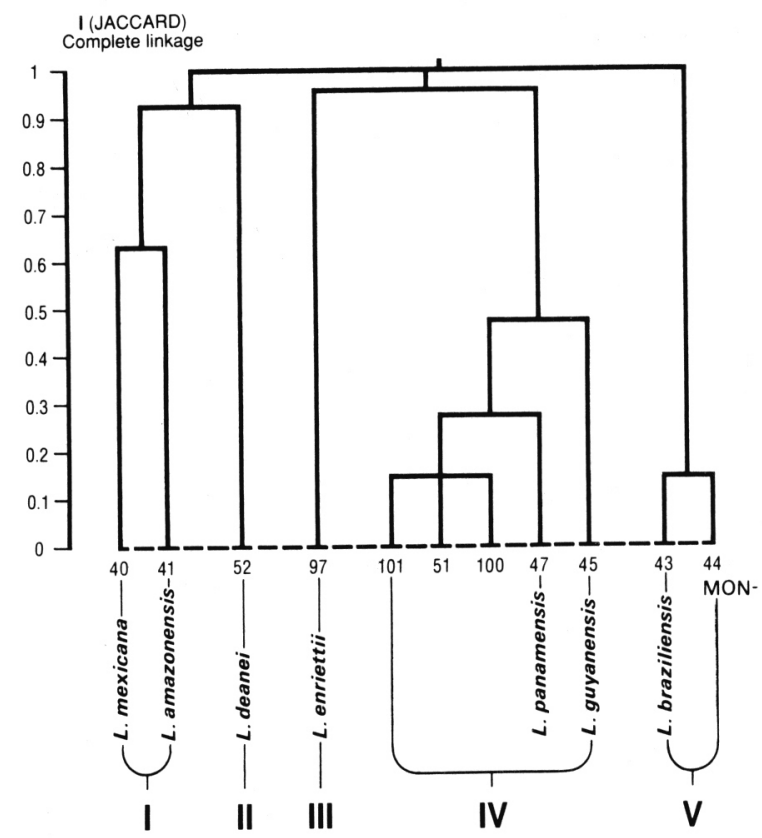

Fig. 4. - Phenogram of New World Leishmania created using 11 zymodemes, 13 enzymes and 65 electromorphs. Five main groups are present. Group I formed by species of the Leishmania sub-genus (L. mexicana, L. amazonensis) appears distant from groups IV (L. guyanensis, L. panamensis) and V (L. braziliensis) belonging to the Viannia sub-genus, themselves very distinct. The groups II (L. deani) and III (L. enriettii), isolated from Vertebrates other than Man, are linked to other groups (respectively group I and group IV) at a very low level of affinity, which does not allow a valid confirmation of the clustering.

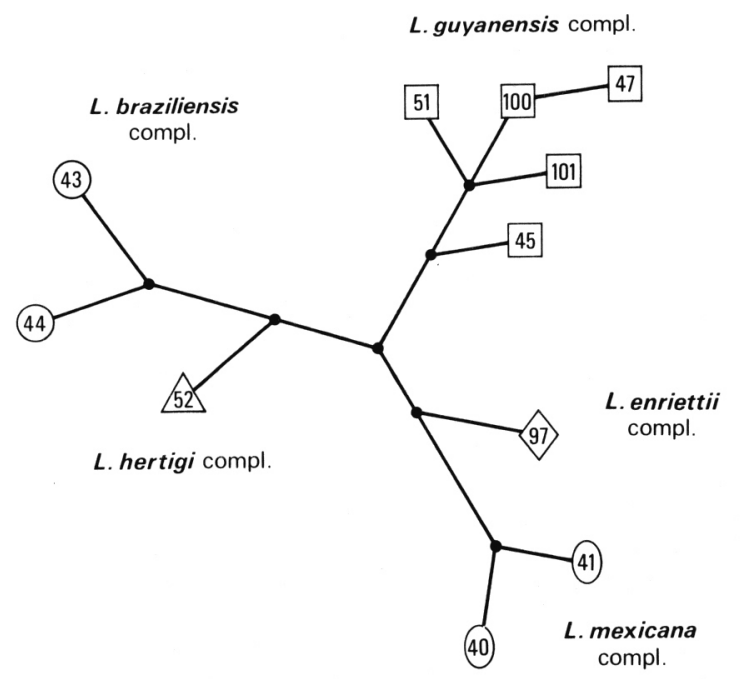

FIG. 5. - Network constructed using the same data than the phenogram of figure 4. The little number of zymodemes and the rare electromorphs in common between the different complexes ( 1 to $3 / 13)$ do not allow a great precision regarding their relative positions. Nevertheless, a unique shortest network can be constructed showing three main branches. first construct a network whose center holds the common ancestor or a nearby unit (fig. 5). The construction of the definitive cladogram is performed by optimization. The most parsimonious tree, issued from the most parsimonious network, $i$. $e$. the construction containing the least number of reversions, is the one selected $(56,60)$. Its congruence with other classification systems, e. g. Linnean systems, further supports its usefulness. The advantage of the cladistics is to maintain character states until the end of the construction and thus to test their phylogenetic value. It is recommended to superimpose the character states on to the OTUs and the HTUs so as to test the compatibility of the different enzymatic systems between each other (56); also, it is easier to recognize the pleisiomorph, synapomorph or autapomorph states. Finally, the method is closer than any other to " natural " taxonomy. The correct interpretation of cladograms cannot in fact be made without an in-depth consideration of the group origin and the phylogenetic meaning of its branches. We can thus speak of true evolutionary systematics (31).

- Cladistics and mode of evolution:

clonality or/and sexuality

As mentioned above, the use of zymodemes as independent OTUs presumes the absence, or at least the low frequency of genetic exchange. Whether under haploid or diploid conditions, the rare heterozygotes observed can be considered to be « fixed hybrids » similar to those observed in some multicellular organisms with agamous reproduction (42). In other words, the hypothesis of a clonal evolution constitutes the basis of the numerical classifications used here.

Actually however, the existence of sexual processes in Leishmania now appears plausible. A number of arguments supporting this hypothesis have accumulated in recent years. Thus, in the same geographical area, electromorphs have been found that might reflect a sexuality: for example, in Israel, strains with PGD 110/118, others with PGD 110 and others with PGD 118 (33); in Spain (Catalonia), strains with either NP1 100/140 or NP1 100 or NP1 140 have been isolated from the same small focus (45). Moreover, among these sympatric strains, pulsed field gel electrophoresis has allowed the repeated finding of particular size of chromosomes whose presence might be explained by a segregation process between daughter cells (44). Likewise, a putative natural hybrid of $L$. major and $L$. arabica has recently been reported (10). Finally, a cytogamy phenomenon has been observed in L. infantum and L. tropica (28).

Nevertheless, even if the existence of genetic exchange is confirmed in Leishmania as in the closely related species Trypanosoma brucei (58), it is unlikely that this will greatly affect the cladogram. On the one hand, whether this genetic exchange involves a classical Mendelian sexuality or a conjugation-type parasexuality, it is not certain that, they 
play the same role in evolution in these primitive flagellates as in upper Eukaryotes. On the other hand, the only branches of the cladogram which might be affected are the terminal ones, where "rake" or "network " effects are most often observed. The main branches, characteristic of the complexes, remain remarkably stable in most of the phenetic or cladistic trees. In the hypothesis of a sexual evolution, this stability could be explained by the absence or the rarity of exchanges at this level, due to the ancient nature of species divergence and to the presence of ecological or geographical barriers.

In any case, until further clarification of these points, we have, in our constructions, considered the rare heterozygous structures as autonomous electromorphs.

\section{SUGGESTIONS FOR A NEW CLASSIFICATION OF THE GENUS LEISHMANIA}

\section{1 - MONOPHYLETISM OR POLYPHYLETISM OF THE GENUS Leishmania?}

Even if the reptile parasites (genus Sauroleishmania) are excluded, the use of enzymes in the taxonomy of Leishmania does not allow confirmation or rejection of the monophyletic origin of the group: for the moment, the sub-genera Viannia and Leishmania appear to have few or no isoenzymatic similarities. Moreover, the neotropical complex, L. mexicana (s. g. Leishmania) seems only distantly related to its Old World homologs. At present, the collected data does not allow to question the systematic divisions proposed by Lainson and Shaw (25). Having essentially studied the Old Word zymodemes (fig. 2 and 3) and very little the New World (fig. 4 and 5), we have not yet attempted to construct a phyletic tree including zymodemes of both Worlds.

\section{2 - RETURN TO BINOMINAL NOMENCLATURE} AND THE NOTION OF PHENETIC COMPLEXES

Since the 1984 International Symposium on Leishmania (46), the scientific community appears to agree to rehabilitate the binominal nomenclature (16). This position appears to be well adapted to the taxonomical situation of Leishmania: in the absence of evidence for a classical Mendelian system in the genus, it avoids any formalisation in that sense, notably by doing away with the notion of sub-species. Moreover, the category « species» can be used without giving to this word the value of mixiological biological species. It should be noted that many animal or plant species known as sexual are in the same situation, due to the lack of sufficient genetic analysis and detailed crossing experiments.

The term " leishmanial complex ", introduced by Lainson and Shaw (23), in the Linnean sense of groups of species or sub-species, is reused here in its Sokalian phenetic sense, that of group of OTUs. According to our definition, the phenetic complexes are made of closely related zymodemes, $i$. e. zymodemes both sufficiently linked together and sufficiently separated from the other clusters to form " true " groups. The technique of moving centres (strong forms) and the correspondence analysis make it possible to confirm this relationship. At this stage, cladistics adds its contribution: the complexes then appear as autonomous phyla due to strong synapomorphies.

Once these precisions have been added, the reintegration of the old binominal classification can be made simply through the heads of new complexes. The corresponding reference strains (50) act as enzymatic markers. For example, L. infantum Nicolle, 1908, zymodeme MON-1, strain from Tunis (MHOM/TN/80/IPT1) becomes the marker for the L. infantum complex.

As we have emphasized, in the genus Leishmania the monothetic Linnean method is not recommended to individualize and a fortiori to describe a taxon whose level is less than that of the sub-genus. The fragility of extrinsic characters should be stressed, whether they are clinical, epidemiological or biogeographic. Thus, in the Mediterranean basin, the $L$. infantum complex groups dermotropic and viscerotropic zymodemes. Moreover, one of them, MON-1, has been found to be responsible for cutaneous and visceral forms (48). Likewise, the Latin American taxon L. chagasi Cunha and Chagas, 1937 cannot be distinguished from L. infantum MON-1 on the basis of its isoenzyme profile (41).

As recommended at the 1984 Symposium (46), numerical taxonomy demands a high degree of rigidity, notably:

« 5.3.3. At the time of first publication, the diagnosis must be accompanied by designation of the geographical locality of isolation (" type locality ") and the original host (" type host ") and by designation of the deposition in a recognized bank of one, and only one reference strain.

5.3.4. It is a wise precaution to send this strain or, if possible, a clone of this strain, to all the cryobanks likely to be able to protect and preserve it. Homologous strains from the same locality and the same host, if they exist, should also be deposited.

5.4. In the case of an old taxon for which no live material exists, it is recommended that a new strain be officially designated. This strain should correspond to the geographical, epidemiological and clinical characteristics of the original description ».

In addition, special attention should be paid to priority rules. For example, enzymatic analyses have shown that L. guyanensis Floch, 1954 and L. panamensis Lainson and Shaw, 1972 zymodemes are phenetically related and, on the contrary, are both different from L. braziliensis Vianna, 1911 (40). If a new phenetic complex is proposed to regroup, L. guyanensis and L. panamensis, it would be 
necessary to use L. guyanensis as head of the new complex. In any other case, the "inventor" is a free man and systematics is an open science (46).

\section{3 - The Sandfly-Leishmania « COUPle ».}

CONGRUENCE OF BOTH SYSTEMATICS

For the past several years, biochemical techniques for Leishmania identification have greatly advanced the screening of «true vectors ». In the Old World, enzymatic typing of strains isolated from natural sanfly populations has led to some suspected vectors being shown not to be true vectors. But without a doubt the most important aspect of this research is the proof of a high correlation between leishmanial complexes and the sub-genera of the genus Phlebotomus. Thus, all the zymodemes of L. major isolated until now were so from the sub-genus Phlebotomus (P. duboscqui, P. papatasi). The same observation can be made for $L$. infantum, isolated from the sub-genus Larroussius * (P. ariasi, P. perfiliewi, P. perniciosus) and for L. tropica always isolated from Paraphlebotomus (P. sergenti s. 1.). In phyletic terms, this observation leads to the conclusion that the parasite's evolution has generally followed a parallel pathway to that of the vector (coevolution) (18). Thus, this sanfly-Leishmania correlation strengthens the value of the dendrogram made from units obtained from intrinsic characters, $i$. e. the zymodeme.

\section{4 - Mixed Linnean-Adansonian Classification}

Bringing together the dual Linnean-Adansonian approaches suggested throughout this paper, we propose a new classification.

The classification remains Linnean for the higher levels in the taxonomic hierarchy, from kingdom to sub-genus. The amended genus Leishmania Ross, 1903 no longer includes the sub-genus Sauroleishmania, raised to the genus level (19). It is sub-divided, however, into two sub-genera, Leishmania Ross, 1903 and Viannia Lainson and Shaw, 1987.

The classification becomes Adansonian in so far as binomials, today revalidated, are used only to " mark " complexes which now proceed from numerical analysis. Subspecific trinomials are naturally eliminated. Finally, the concept of complex is not used in the Linnean sense of species or sub-species groups, but in the Sokalian sense

\footnotetext{
* An exception concerns L. infantum MON-1 transmitted in Latin America by Lutzomyia longipalpis under the binomial L. chagasi. In the hypothesis of a transoceanic migration of $L$. infantum, this would be an example of «capture » phenomenon.
}

of sub-groups of OTUs, here zymodemes. Any complex must bear the name of its " marker " binomial when one exists. If not, the oldest zymodeme is used. It is thus raised to the level of a «species» and described as a new binomial.

\section{$* *$}

In conclusion, we want to briefly recall the spirit of the proposed classification:

The new taxonomy uses enzymatic criteria and is based on numerical techniques for taxonomic units up to but excluding the sub-genus. The characters (or the character states) are represented by electromorphs, $i$. e. isoenzymes. They are considered simultaneously at every partition level. The point of departure is the zymodeme (OTU), which is made up of a group of strains with the same enzymatic profile. From this point on, the binomial loses its biological significance and is only applicable to a particular zymodeme, a simple elementary unit whose essential role is to act as a marker for a complex. In the phenetic approach, the aggregation of zymodemes and zymodeme clusters up to the complex is performed using a so-called agglomerative hierarchical non-overlapping clustering technique.

The phylogenetic reconstruction (4) is performed using the cladistic method. Here the isoenzymes are character states of the character represented by the enzyme. In practice, this system uses the same OTUs and demands the prior elaboration of networks (60). Wherever possible, the cladistic technique should be used. It takes into account the phylogenetic significance of character states and units better than any other method. In particular, it makes it possible to think in terms of evolution, $i$. $e$. in terms of genesis and separation of different taxonomic levels.

Above the complex, the traditional categories are once again used: $(i)$ that of sub-genus to designate parasites having a suprapyloric (Leishmania) or peripyloric (Viannia) development in the vector; (ii) that of genus (Leishmania) to designate the Trypanosomatidae of mammals having a dimorphous cycle: amastigote in reservoir, promastigote in vector.

\section{A REVISED CLASSIFICATION OF THE WORLD Leishmania}

Sub-Kingdom Protozoa Goldfuss, 1817.

Phylum Sarcomastigophora Honigberg and Balamuth, 1963.

Sub-Phylum Mastigophora Diesing, 1886.

Class Zoomastigophores Calkins, 1909.

Order Kinetoplastida Honigberg, 1963.

Sub-Order Trypanosomatina Kent, 1880.

Family Trypanosomatidae Döflein, 1901. 
Genus Leishmania Ross, 1903.

A. Sub-genus Leishmania Ross, 1903.

1. L. donovani phenetic Complex ${ }^{1}$ (13 zymodemes MON-).

. L. donovani (Laveran and Mesnil, 1903) ${ }^{2}$. Locus classicus India.

L. archibaldi Castellani and Chalmers, 1919. Loc. class. Sudan.

2. L. infantum phenetic Complex (18 zymodemes MON-).

. L. infantum Nicolle, 1908. Loc. class. Tunisia (syn. L. chagasi Cunha and Chagas, 1937).

3. L. tropica phenetic Complex (28 zymodemes MON-).

. L. tropica (Wright, 1903). Loc. class. USSR.

L. killicki Rioux, Lanotte and Pratlong, 1986. Loc. class. Tunisia.

4. L. major phenetic Complex (12 zymodemes MON-). . L. major Yakimoff and Schokhor, 1914. Loc. class. Turkestan, USSR.

5. L. gerbilli phenetic Complex (1 zymodeme MON-).

. L. gerbilli Wang, Qu and Guan, 1973. Loc. class. China.

6. L. arabica phenetic Complex (1 zymodeme MON-). . L. arabica Peters, Elbihari and Evans, 1986. Loc. class. Saoudi Arabia.

7. L. aethiopica phenetic Complex (3 zymodemes MON-).

. L. aethiopica Bray, Ashford and Bray, 1973. Loc. class. Ethiopia.

8. L. mexicana phenetic Complex ${ }^{3}$ (3 zymodemes MON-).

. L. mexicana Biagi, 1953. Loc. class. Mexico.

L. amazonensis Lainson and Shaw, 1972. Loc. class. Brazil. (syn. L. garnhami Scorza et al., 1979).

L. venezuelensis Bonfante-Garrido, 1980. Loc. class. Venezuela.

9. L. enriettii phenetic Complex (1 zymodeme MON-).

. L. enrietii Muniz and Medina, 1948. Loc. class. Brazil.

1. As said in the text, the term complex is used here in the sense of a subset of OTUs. The L. gerbilli, L. arabica and $L$. enriettii phenetic complexes formed by only one unit are considered as subsets of the type single. Although fundamental differences exist between the notion of complex as used here and that established by Lainson and Shaw, 1972, a congruence of the two classifications should be noted.

2. First species described in the complex. L. donovani is also the type species of nominal genus Leishmania and of nominal sub-genus Leishmania.

3. According to Lainson and Shaw, 1979, L. pifanoi Medina and Romero, 1959 and L. aristedisi Lainson and Shaw, 1979, should be put in the L. mexicana complex. We did not have the opportunity to study them enzymatically.
10. L. hertigi phenetic Complex (2 zymodemes MON-).

. L. hertigi Herrer, 1971. Loc. class. Panama.

L. deanei Lainson and shaw, 1977, Loc. class. Brazil.

B. Sub-genus Viannia Lainson and Shaw, $1987^{4}$.

11. L. braziliensis phenetic Complex (2 zymodemes MON-).

- L. braziliensis Viannia, $1911^{5}$. Loc. class. Brazil.

L. peruviana Velez, 1913. Loc. class. Peru.

12. L. guyanensis phenetic Complex (5 zymodemes MON-).

. L. guyanensis Floch, 1954. Loc. class. French Guyana.

L. panamensis Lainson and Shaw, 1972. Loc. class. Panama.

Acknowledgments. - Our sincere thanks are due to Doctors R. W. AshFord, D. M. JARRY, R. Killick-Kendrick, R. Lainson and R. D. WARD for valuable advice.

\section{REFERENCES}

1. Adanson M. : Famille naturelle des Plantes. Vincent, Paris, $1763, I, 419 \mathrm{p}$.

2. Al-Hussayni N. K., Rassam M. B., Jawdat S. Z., Wahid F. N. : Numerical taxonomy of some Old World Leishmania species. Trans. R. Soc. Trop. Med. Hyg., 1987, 81, 581-586.

3. Angelici M. C., Gramiccia M., Gradoni L. : Study on genetic polymorphism of Leishmania infantum through the analysis of restriction enzyme digestion patterns of kinetoplast DNA. Parasitology, 1989, 99, 301-309.

4. Avise J. C. : Protein variation and phylogenetic reconstruction. In: Protein polymorphism. Adaptative and taxonomic significance (Rollison D., ed.). Oxford G. S., 1983, 24, 103-130.

5. Barker D. C., Butcher J., Gibson L. J., Kennedy W. P. K., Williams R. H., Cuba Cuba C. A., Marsden P. D., Lainson R. Shaw J. J. : Sequence homology of kinetoplast DNA in Leishmania studied by filter hybridization of endonuclease digested fragments and in situ hybridization of individual organisms. In: Leishmania. Taxonomie et phylogenèse (Rioux J. A., ed.). IMEEE, Montpellier, 1986, 41-55.

6. Beverley S. M., Ismach R., McMahon-Pratt D. : Evolution of the genus Leishmania Ross, 1903 as revealed by comparisons of nuclear DNA restriction fragment patterns. In: Leishmania. Taxonomie et phylogenèse (Rioux J. A., ed.). IMEEE, Montpellier, 1986, 265-267.

7. Chance M. L. : The nomenclature of enzyme variants with particular reference to parasitic protozoa. In: Leishmania. Taxonomie et phylogenèse (Rioux J. A., ed.). IMEEE, Montpellier, 1986, 85-89.

4. Three new species have recently been described in this subgenus, but we did not have the opportunity to study them enzymatically. Therefore, they are not (yet) included in this classification. They are: L. lainsoni Silveira, Shaw, Braga and Ishikawa, 1987. Loc. class. Brazil (55), L. naiffi, Lainson and Shaw, 1989. Loc. class. Brazil (26) and L. shawi Lainson, Braga, de Souza, Povoa, Ishikawa and Silveira, 1989. Loc. class. Brazil (22).

5. Type species of nominal sub-genus Viannia. 
8. Chance M. L., Peters W., Schchory L. : Biochemical taxonomy of Leishmania. I. Observations on DNA. Ann. Trop. Med. Parasitol., 1974, 68, 307-316.

9. Chardy P. : L'espèce et la taxonomie numérique. In: Les problèmes de l'espèce dans le règne animal. Mém. Soc. Zool. Fr., 1980, 40, 65-97.

10. Evans D. A., Kennedy W. P. K., Elbihari S., Chapman C. J., Smith V., Peters W. : Hybrid formation within the genus Leishmania? Parassitologia, 1987, 29, 165-173.

11. Gardener P. J., Chance M. L., Peters W. : Biochemical taxonomy of Leishmania. II. Electrophoretic variation of malade dehydrogenase. Ann. Trop. Med. Parasitol., 1974, 68, 317-325.

12. Gardener P. J., Howells R. E. : Isoenzyme variation in leishmanial parasites. J. Protozool., 1972, 19, 47.

13. Giannini S. H., Schittini M., Keithly J. S., Warburton P. W., Cantor C. R., Van Der Ploeg L. H. T. : Karyotype analysis of Leishmania species and its use in classification and clinical diagnosis. Science, 1986, 232, 762-765.

14. Godfrey D. G. : The zymodemes of trypanosomes. In: Problems in the identification of parasites and their vectors. Symp. Brit. Soc. Parasitol., 1979, 17, 31-53.

15. Goujet D., Matile L., Janvier P., Hugot J. P. : Systématique cladistique. In: Biosystema. Soc. Fr. System., 1988, 2, 195 p.

16. Grimaldi G. Jr., Tesh R. B., McMahon-Pratt D. : A review of the geographic distribution and epidemiology of leishmaniasis in the New World. Am. J. Trop. Med. Hyg., 1989, 41, 687-725.

17. Hennnig W. : Phylogenetic systematics. Ann. Rev. Entomol., 1965, 10, 97-116.

18. Killick-Kendrick R. : Some epidemiological consequences of the evolutionary fit between Leishmania and their Phlebotomine vectors. Bull. Soc. Path. Exot., 1985, 78, 747-755.

19. Killick-Kendrick R., Lainson R., Rioux J. A., Saf'Janova W. M. : The taxonomy of Leishmania-like parasites of Reptiles. In: Leishmania. Taxonomie et phylogenèse (Rioux J. A., ed.). IMEEE, Montpellier, 1986, 143-148.

20. Kreutzer R. D., Christensen H. A. : Characterization of Leishmania spp. by isoenzyme electophoresis. Ann. J. Trop. Med. Hyg., 1980, 29, 199-208.

21. Kreutzer R. D., Semko M. E., Hendricks L. D., Wright N. : Identification of Leishmania spp. by multiple isozyme analysis. Am. J. Trop. Med. Hyg., 1983, 32, 703-715.

22. Lainson R., Braga R. R., de Souza A. A. A., Povoa M. M., Ishikawa E. A., Silveira F. T. : Leishmania (Vianna) shawi sp. n., a parasite of monkeys, cloths and procyonids in Amazonian Brazil. Ann. Parasitol. Hum. Comp., 1989, 64, 200-207.

23. Lainson R., Shaw J. J. : Leishmaniasis of the New World: taxonomic problems. Brit. Med. Bull., 1972, 28, 44-48.

24. Lainson R., Shaw J. J. : The role of animals in the epidemiology of South American leishmaniasis. In: Biology of the Kinetoplastida (Lumsden W. H. R., Evans D. A., eds.). Academic Press, London, 1979, 2, 1-116.

25. Lainson R., Shaw J. J. : Evolution, classification and geographical distribution. In: The leishmaniases in biology and medicine (Peters W., Killick-Kendrick R., eds.). Academic Press, London, 1987, 1, 1-120.

26. Lainson R. R., Shaw J. J. : Leishmania (Viannia) naiffi sp. n., a parasite of the armadillo, Dasypus novemcinctus (L.) in Amazonian Brazil. Ann. Parasitol. Hum. comp., 1989, 64, 3-9.

27. Lanham S. M., Grendon J. M., Miles M. A., Povoa M M., Almeida de Souza A. A. : A comparison of electrophoretic methods for isoenzyme characterization of trypanosomids. I. Standard stocks of Trypanosoma cruzi zymodemes from northeast Brazil. Trans. R. Soc. Trop. Med. Hyg., 1981, 75, 742-750.

28. Lanotte G., Rioux J. A. : Fusion cellulaire chez les Leishmania (Kinetoplastida, Trypanosomatidae). C. R. Acad. Sci., Paris, 1990, 310, sér. III, 285-288.
29. Lanotte G., Rioux J. A., Lepart J., Maazoun R., Pasteur N., Pratlong F. : Contribution de la cladistique numérique à la phylétique du genre Leishmania Ross, 1903 (Kinetoplastida, Trypanosomatidae). Utilisation des caractères enzymatiques. C. R. Acad. Sci., Paris, 1984, 299, 769-772.

30. Lanotte G., Rioux J. A., Maazoun R., Pasteur N., Pratlong F., Lépart J. : Application de la méthode numérique à la taxonomie du genre Leishmania Ross, 1903. A propos de 146 souches originaires de l'Ancien Monde. Utilisation des allozymes. Corollaires épidémiologiques et phylétiques. Ann. Parasitol. Hum. Comp., 1981, 56, 575-592.

31. Lanotte G., Rioux J. A., Serres E. : Approche cladistique du genre Leishmania Ross, 1903. A propos de 192 souches de l'Ancien Monde. Analyse numérique de 50 zymodèmes identifiés par 15 enzymes et 96 isoenzymes. In: Leishmania. Taxonomie et phylogenèse (Rioux J. A., ed.). IMEEE, Montpellier, 196, 269-288.

32. Le Blancq S. M., Cibulkis R. E., Peters W. : Leishmania in the Old Wolrd. 5. Numerical analysis of isoenzyme data. Trans R. Soc. Trop. Med. Hyg., 1986, 80, 517-524.

33. Le Blancq S. M., Schnur L. F., Schlein Y. : An apparent association of enzymic variants of Leishmania major with specific geographical areas in Istrael. Bull. Soc. Path. Exot., 1983, 76, 543-548.

34. Legendre L., Legendre P. : Écologie numérique. Coll. d'Écologie. Masson, Paris, 1979, 13, 2 vol., 198 p. et 248 p.

35. Linne C. : Philosophia botanica. Gleditsch, 1780, $361 \mathrm{p}$.

36. Maazoun R., Lanotte G., Pasteur N., Rioux J. A., Kennou M. F., Pratlong F. : Écologie des leishmanioses dans le Sud de la France. 16. Contribution à l'analyse chimiotaxonomique des parasites de la leishmaniose viscérale méditerranéenne. A propos de 55 souches isolées en Cévennes, Côte d'Azur, Corse et Tunisie. Ann. Parasitol. Hum. Comp., 1981, 56, 131-146.

37. Maazoun R., Lanotte G., Rioux J. A., Pasteur N., KillickKendrick R., Pratlong F. : Signification du polymorphisme enzymatique chez les Leishmanies. A propos de trois souches hétérozygotes de Leishmania infantum Nicolle, 1908, Leishmania cf. tarentolae Wenyon, 1921 et Leishmania aethiopica Bray, Ashford et Bray, 1973. Ann. Parasitol. Hum. Comp., 1981, 56, 467-475.

38. Mayr E. : Principles of Systematic Zoology. McGraw-Hill, New York, 1969, $428 \mathrm{p}$.

39. McMahon-Pratt D., David J. R. : Monoclonal antibodies that distinguish between New World species of Leishmania. Nature, 1981, 291, 581-583.

40. Moreno G., Pratlong F., Velez I. D., Restrepo M., Rioux J. A. : Individualisation du complexe Leishmania guyanensis. A propos de l'analyse numérique de sept zymodènes. In: Leishmania. Taxonomie et phylogenèse (Rioux J. A., ed.). IMEEE, Montpellier, 1986, 165-172.

41. Moreno G., Rioux J. A., Lanotte G., Pratlong F., Serres E. : Le complexe Leishmania donovani s. 1. Analyse enzymatique et traitement numérique. Individualisation du complexe Leishmania infantum. Corollaires biogéographiques et phylétiques. A propos de 146 souches originaires de l'Ancien et du Nouveau Monde. In: Leishmania. Taxonomie et phylogenèse (Rioux J. A., ed.). IMEEE, Montpellier, 1986, 105-117.

42. Nei M. : Molecular population genetics and evolution. In: Frontiers of biology. North-Holland Publ. Co., Amsterdam, 1975, $288 \mathrm{p}$.

43. OMS : Lutte contre les leishmanioses. Série de rapports techniques. OMS, Genève, 1990, 793, $176 \mathrm{p}$.

44. Pagès M., Bastien P., Veas F., Rossi V., Bellis M., Wincker P., Rioux J. A., Roizès G. : Chromosome size and number polymorphisms in Leishmania infantum suggest amplification/deletion and possible genetic exchange. Mol. Biochem. Parasitol., $1989,36,161-168$. 
45. Piarroux R. : Intérêt de l'isoélectrofocalisation dans l'analyse isoenzymatique du genre Leishmania Ross, 1903. A propos de 25 souches de l'Ancien Monde. Diplôme d'Études Approfondies, Université Montpellier I, $30 \mathrm{p}$.

46. Rioux J. A. : Leishmania. Taxonomie et phylogenèse. Applications éco-épidémiologiques (Rioux J. A., ed.). IMEEE, Montpellier, 1986, $537 \mathrm{p}$.

47. Rioux J. A., Lanotte G., Maazoun R., Pasteur N. : L'électrophorèse des enzymes dans le genre Leishmania Ross, 1903. Parassitologia, 1985, 27, 141-156.

48. Rioux J. A., Lanotte G., Petter F., Dereure J., Akalay O., Pratlong F., Velez I. D., Fikri N. B., Maazoun R., Denial M., Jarry D. M., Zahaf A., Ashford R. W., Cadi-Soussi M., Killick-Kendrick R., Benmansour N., Moreno G., Perieres J., Guilvard E., Zribi M., Kennou M. F., Rispail P., Knechli R., Serres E. : Les leishmanioses cutanées du bassin Méditerranéen occidental. De l'identification enzymatique à l'analyse éco-épidémiologique. L'exemple de trois « foyers », tunisien, marocain et français. In : Leishmania. Taxonomie et phylogénèse (Rioux J. A., ed.). IMEEE, Montpellier, 1986, 365-396.

49. Rioux J. A., Lanotte G., Pratlong F. : Leishmania killicki n. sp. (Kinetoplastida, Trypanosomatidae). In: Leishmania. Taxonomie et phylogenèse (Rioux J. A., ed.). IMEEE, Montpellier, 1986, 139-142.

50. Rioux J. A., Lanotte G., Pratlong F., Martini A., Serres E., Belmonte A. : Centre international de cryoconservation, d'identification enzymatique et d'étude taxonomique des Leishmania (Montpellier, France). Structure et fonctionnement. In: Leishmania. Taxonomie et phylogenèse (Rioux J. A., ed.). IMEEE, Montpellier, 1986, 485-502.

51. Roux M. : Algorithmes de classification. Masson, Paris, 1985, $147 \mathrm{p}$.
52. Schnur L. F., Chance M. L., Ebert F., Thomas S. C., Peters W. : The biochemical and serological taxonomy of visceralizing Leishmania. Ann. Trop. Med. Parasitol., 1981, 75, 131-144.

53. Schnur L. F., Zuckerman A., Greenblatt M. D. : Leishmanial serotypes as distinguished by the gel diffusion of factors excreted in vitro and in vivo. Isr. J. Med. Sci., 1972, 8, 932-942.

54. Serres E., Roux M. : Pratique de la classification automatique. L'exemple des Leishmania. In: Leishmania. Taxonomie et phylogenèse (Rioux J. A., ed.). IMEEE, Montpellier, 1986, 27-40.

55. Silveira F. T., Shaw J. J., Braga R. R., Ishikawa E. : Dermal leishmaniasis in the Amazon region of Brazil: Leishmania (Viannia) lainsoni sp. n., a new parasite from the State of Parà. Mem. Inst. Oswaldo Cruz., 1987, 82, 289-291.

56. Sneath P. H. A., Sokal R. R. : Numerical taxonomy. The principles and practice of numerical classification. Freeman W. H., San Francisco, 1973, 571 p.

57. Sokal R. R., Sneath P. H. A. : Principles of Nưmerical Taxonomy. Freeman $W . W$. , San Francisco, 1963, 359 p.

58. Sternberg J., Turner C. M. R., Wells J. M., RandfordCartwright L. C., Le Page R. W. F., Tait A. : Gene exchange in African trypanosomes: frequency and allelic segregation. Mol. Biochem. Parasitol., 1989, 34, 269-280.

59. Tibayrenc M., Cariou M. L., Solignac M. : Interprétation génétique des zymogrammes de Flagellés des genres Trypanosoma et Leishmania. C. R. Acad. Sci., Paris, 1981, 292, 623-625.

60. Wagner W. H. : Problems in the classification of ferns. In: Recent advances in botany. University Toronto Press, Montréal, 1961, 841-844. 